\title{
Conocimiento y centralidad: el papel de los expertos en las redes de discusión política
}

\author{
Livia García Faroldi - Universidad de Málaga ${ }^{1}$
}

\section{Resumen}

En esta investigación se ha estudiado el proceso de influencia interpersonal en las redes de discusión política, una variable relevante que debe tener en cuenta cualquier agente social situado en las esferas del Estado, del mercado o de la sociedad civil. Se analiza el papel desempeñado por los "expertos" sobre la Unión Europea (detectados a través de dos escalas creadas para la ocasión) en estas redes de discusión, el lugar que ocupan en estas redes y si se encuentran en las posiciones más centrales de la estructura social, localizaciones definidas a través de una versión actualizada de la escala de posición social sugerida por Galtung en los años 60.

Palabras clave: Redes de discusión política - Liderazgo de opinión - Difusión de innovaciones - Escala de posición social de Galtung.

\begin{abstract}
This research studies the process of interpersonal influence that takes place in political discussion networks, an important factor that must be taken into account by any social agent in State, market or civil society spheres. We analize the role of "experts" on European Union in these networks (detected through two scales created for this study), the location they occupy in these networks and if they are situated in most central positions in the social structure, defining these locations with a modern version of the social positions scale suggested by Galtung in the sixties.
\end{abstract}

Key words: Political discussion networks - Opinion leadership - Diffusion of innovations - Social positions Galtung's scale.

\section{I ntroducción}

España es, desde hace poco más de dos décadas, miembro de la Unión Europea (llamada entonces Comunidad Económica Europea). Tras la incorporación, los ciudadanos desarrollan progresivamente una actitud hacia esta nueva realidad política (favorable o no) y, en su caso, una identificación con este nuevo ámbito territorial del que forman parte. Desde las instituciones estatales y las comunitarias, se han desarrollado diversas políticas para fomentar las actitudes favorables a la Unión y la identidad europea. En esta investigación se considera que ambos fenómenos son nuevas ideas que se difunden progresivamente en la sociedad española. En todo proceso de difusión existen actores que adoptan

\footnotetext{
${ }^{1}$ Enviar correspondencia a: Igarcia@uma.es
} 
primero la innovación y animan al resto a seguir sus pasos. Los estudios sobre sociología política y los análisis reticulares que estudian cómo se difunde una innovación en una comunidad prestan atención al liderazgo ejercido por ciertos actores, individuos que tienen un mayor interés, conocimiento e implicación en el campo que lideran y que, precisamente por ello, son reconocidos por el resto de los miembros del grupo como personas a las que pedir información y/o consejo. En este contexto, las redes de discusión sobre política se convierten en un lugar privilegiado para estudiar cómo los ciudadanos crean, modifican y adaptan sus actitudes y creencias sobre los diversos contenidos de la esfera pública.

Los agentes sociales (Estado, organizaciones, asociaciones, etc.) deben tener en cuenta a estos actores al planificar sus políticas para que éstas lleguen de manera efectiva a toda la población, ya que estos individuos muestran un mayor interés por y una más elevada participación en la esfera pública. En la investigación que se ha desarrollado se supone que las personas más familiarizadas con la Unión adoptan antes una actitud a favor de la UE y una identidad europea y que ocupan posiciones centrales en las redes de discusión política de las que forman parte. Para ello, se han creado dos escalas que miden el grado en que una persona es experta en asuntos comunitarios, las escalas de experto práctico y teórico.

\section{Marco teórico}

\section{Los estudios sobre el liderazgo de opinión en los procesos de influencia interpersonal}

Uno de los ámbitos de interés en las diferentes disciplinas sociales (desde la psicología social a la sociología política) y diferentes enfoques metodológicos (el reticular y el individualista o "atomista") es la importancia de tener en cuenta el liderazgo ejercido por ciertos actores para estudiar los procesos de influencia interpersonal. En los años cuarenta, los hoy clásicos estudios de Lazarsfeld, Berelson, Katz y otros autores sobre los factores que influyen en la decisión del voto llaman la atención sobre un fenómeno repetidamente comprobado en estudios posteriores: la existencia de unos líderes de opinión "horizontales", lo que dio lugar a la llamada "hipótesis del flujo de comunicación en dos pasos". De esta manera se supera la teoría de "aguja hipodérmica" que suponía un efecto directo e inmediato de los medios de comunicación (prensa y radio en aquella época) sobre una audiencia pasiva. 
Como el propio Lazarsfeld reconoce, el descubrimiento de líderes que influyen en su entorno inmediato fue casi accidental. Durante la campaña presidencial estadounidense de 1940, Lazarsfeld, Berelson y Gaudet (1954) investigaron los efectos de la prensa y la radio en la decisión del voto en una comunidad de Ohio. Los datos mostraron que los efectos de los medios de comunicación eran pequeños en comparación con el papel jugado por las influencias personales. Concluyeron que los votantes se deciden ajustándose al clima político de su entorno social. El hallazgo fue relevante ya que, hasta entonces, se había asumido que las opiniones se formaban en la elite de la comunidad y descendían hacia el resto de los estratos sociales, se producía un liderazgo "vertical" de la cúspide hacia la base. Esta investigación mostró que existía también un liderazgo de opinión "horizontal" pues, como los propios autores comentaban, estos líderes de opinión "no son idénticos a los individuos socialmente prominentes en la comunidad, ni a los individuos más ricos, ni a los líderes cívicos" (p.50). ${ }^{2}$

Se formuló así la hipótesis de que existía un flujo en dos pasos en la comunicación (two-step flow of communication) entre los medios de masas y el público: en cada estrato social, los líderes de opinión tenían una mayor probabilidad de exponerse a los medios y éstos, a su vez, transmitían esta información al resto de las personas. Las ideas, por tanto, fluyen de la radio y la prensa hasta los líderes de opinión y de éstos a los sectores menos activos de la población. Estos líderes son definidos por los autores como aquellos individuos que se caracterizan por estar muy implicados e interesados por el tema y dotados de más conocimientos sobre el mismo, que actúan como mediadores entre los medios de comunicación y el resto de los individuos, que están menos interesados o tienen una menor participación en la campaña electoral.

Pese a que estos autores no adoptan el análisis de redes, sus reflexiones inciden en la relevancia de las relaciones personales, pues también señalaron que, al margen y por encima de estos líderes "horizontales", existen una serie de interacciones recíprocas entre los componentes del grupo que refuerzan las actitudes todavía no concretadas de cada persona. La distribución de opiniones y actitudes articuladas cristaliza sobre la base de estas interacciones. Por lo tanto, el proceso de formación de la opinión pública no es resultado de los individuos considerados aisladamente, sino que deriva de las interacciones entre los miembros de la sociedad. Los efectos de los medios de comunicación, que era el foco de interés de estos estudiosos, sólo

\footnotetext{
${ }^{2}$ Traducción propia.
} 
pueden comprenderse teniendo en cuenta estas relaciones entre los destinatarios de la comunicación.

En un estudio posterior, Lazarsfeld y Berelson, esta vez junto a McPhee (1963), profundizaron en el estudio de este fenómeno, investigando cómo decidían su voto los ciudadanos de Elmira, una ciudad del Estado de Nueva York, en la campaña presidencial de 1948. Comprobaron que el contexto social más próximo de un sujeto es homogéneo políticamente y que se mantiene así gracias a la discusión política con la familia y los amigos y compañeros, por lo que concluyeron que, en gran medida, la discusión política se mantiene dentro de los grupos más que entre ellos. Se preguntó al entrevistado en quién "delega" 3 el votante la responsabilidad de la discusión política en la comunidad. Como se había observado en sus investigaciones anteriores, el liderazgo recae principalmente en personas similares al votante, son calificados de influyentes cercanos. De hecho, Berelson y sus colegas prefieren, en vez de referirse a líderes de opinión, hablar de la existencia de una compleja red de relaciones de liderazgo: ello es así porque, aunque se pueden diferenciar personas que tienen más probabilidad de estar en el centro de esta red y calificarlos como líderes, también es cierto que dichos influyentes, por su parte, piden consejo sobre política con mayor frecuencia que el resto de la población. Tres son las cualidades que distinguen a estos líderes informales: 1) Un mayor interés y competencia en la esfera de discusión que lideran; 2) una mayor interacción a través de localizaciones sociales más estratégicas y 3) los líderes informales representan o simbolizan las normas dadas del grupo en una particular esfera, precisamente la esfera en la que son influyentes. Es probable que sean activos en la vida social rutinaria, que sean competentes en política, pero siguen siendo ciudadanos ordinarios y por ello son modelos o prototipos para aquellos a los que informa, con los que discute y a los que influye. Se trata de distinguirse un poco sin ser demasiado distinto.

Las conclusiones de estos autores coinciden con las extraídas por los investigadores de redes. El liderazgo de opinión ha sido un fenómeno estudiado especialmente por aquellos que han analizado cómo se difunde una innovación. Un repaso de esta tradición investigadora la realiza Valente (1995). Este autor centra la atención en tres investigaciones sobre difusión de una innovación que recogen conjuntamente datos relacionales y datos sobre el tiempo de adopción: la difusión de una innovación entre un grupo de médicos estadounidenses (Coleman et al, 1966), la

\footnotetext{
${ }^{3}$ Comillas de los autores.
} 
difusión de una semilla híbrida entre campesinos brasileños (Rogers et al, 1970) y la difusión de métodos de planificación familiar entre familias coreanas (Rogers y Kincaid, 1981).

Señala Valente que los líderes son definidos habitualmente como aquellos individuos con mayor número de nominaciones y se supone que tienen una influencia significativa en el ritmo de adopción de una innovación en un determinado grupo social. Una vez se localizaron los líderes, se descubrió que adoptaban más temprano las innovaciones y se postuló que los líderes pasan la información a sus seguidores (se trata precisamente de la hipótesis de la comunicación en dos pasos). Valente considera que, generalmente, los sujetos esperan hasta que los miembros más influyentes del grupo adoptan una innovación. Una vez que la han adoptado, el riesgo y la incertidumbre sobre dicha innovación decrece y es más probable que los seguidores la adopten. El estudio de Coleman mostró que el proceso de difusión ocurría de manera distinta en los doctores: los médicos integrados interactuaban entre sí y se persuadían mutuamente para adoptar, mientras que aquellos aislados no eran influidos por otros miembros del sistema social. Valente analiza los datos de las tres investigaciones y encuentra una correlación moderada entre el liderazgo de opinión (medido por el número de nominaciones recibidas) y el grado de innovación. Sin embargo, de esta asociación no se puede asegurar la causalidad, si el liderazgo influye en la adopción o viceversa. Es más, continúa el autor, la correlación puede ser espuria dado que se tiende a nominar a individuos con un mayor nivel socioeconómico y dicho nivel se asocia con una adopción más temprana.

Valente propone otra forma de aproximación a este asunto. Señala que, aunque no sea una medida del liderazgo de opinión, el número de nominaciones que un individuo envía es una medida rudimentaria del grado de conectividad de un sujeto en la red, ya que indica el grado de actividad de una persona en la comunicación con el resto del sistema social. Las personas muy conectadas reciben la información e influencia sobre una innovación antes que los menos conectados. Por ello, se espera que el grado de conectividad se correlacione positivamente con el grado de innovación, aunque dicha conexión sólo se ha encontrado en el caso de la investigación sobre la difusión de anticonceptivos en las familias coreanas (Rogers y Kincaid, 1981). De hecho, otros estudios sobre difusión muestran que los individuos marginales tienen más probabilidad de adoptar tempranamente la innovación: Menzel (1960), uno de los autores del estudio sobre la innovación médica, argumenta que los marginados están liberados de las normas sociales y por ello 
son capaces de adoptar antes. Además, como las innovaciones son nuevas por definición, a menudo se las percibe como en conflicto o contrarias con las convenciones existentes, así que es difícil que las adopten individuos integrados. La investigación de Becker (1970) muestra que las innovaciones más ventajosas probablemente serán adoptadas en primer lugar por las figuras centrales en la red o por aquellos más nominados, mientras que las innovaciones arriesgadas son adoptadas antes por los miembros marginales, es decir, aquellos que reciben menos nominaciones. Los sujetos más innovadores se contienen a la hora de adoptar una innovación con un alto grado de incertidumbre para mantener o incrementar su liderazgo. En esta investigación se asume que la identidad europea y la actitud favorable a la UE son innovaciones ventajosas $y$, por ello, adoptadas antes por las personas situadas en el centro social. ${ }^{4}$

Volviendo a la sociología americana de mediados del siglo pasado, una última investigación que resulta de interés es la realizada por Merton (1949), en la que descubrió que el consumo de los medios es diferente en las personas llamadas "influyentes" respecto al resto de la población: "para el lector común, la información que encuentra en la revista es una mercancía para consumo personal, ampliando su propio concepto al mundo de los acontecimientos públicos, mientras que para el influyente es una mercancía para cambio, para ser comerciada en busca de un nuevo aumento de prestigio, por permitirle actuar como intérprete de asuntos nacionales e internacionales. Le ayuda a ser un líder de la opinión" (edición 1995, p.475). ${ }^{5}$ Es más, el autor descubrió que algunos influyentes se expresaron sólo en relación con la situación local de su comunidad, mientras otros se refirieron frecuentemente a materias lejanas a ésta. Esta diferencia en la orientación de los influyentes fue la que condujo al autor a distinguir entre los influyentes localistas (o locales) y cosmopolitas. La pertinencia de la distinción entre influyentes cosmopolitas y localistas en esta investigación se debe a que la Unión Europea es, hoy por hoy, una entidad política poco conocida por la mayoría de los ciudadanos, percibida como lejana y poco relevante para la vida cotidiana. Por ello, es probable que las personas busquen consejo o simplemente el contacto con personas más

\footnotetext{
${ }^{4}$ Es probable que estas nuevas ideas sean percibidas de manera diversa por los ciudadanos: las políticas de integración europea no han afectado de igual manera a toda la población y las relaciones entre los diversos niveles identificativos son complejas y dependen de contextos históricos y sociopolíticos. En todo caso, las investigaciones realizadas muestran que las personas con actitudes positivas hacia la Unión y que se identifican en mayor medida con Europa - no sólo en España, sino en otros países miembros- son aquellas con un mayor nivel educativo y socioeconómico luego parece que, más que tratarse de personas aisladas y marginales, son individuos bien situados dentro de su red social y también bien posicionados en la sociedad en general.

${ }^{5}$ Cursivas del autor.
} 
familiarizadas con las instituciones comunitarias, sus funciones y sus modos de funcionamiento y estos conocimientos se adquieren, fundamentalmente, a través de los medios de comunicación. En este ámbito, es razonable pensar que son los influyentes cosmopolitas los que más influencia pueden tener en el resto de su red de contactos.

\section{La relevancia de las posiciones de puente en la difusión de nuevas ideas}

En su conocida teoría de la fuerza de los lazos débiles, Granovetter (1973) destaca la importancia que tienen estos vínculos como puentes, entendiendo por puente el único camino entre dos puntos. Cualquier cosa que quiera ser difundida puede llegar a un gran número de personas y atravesar una gran distancia social cuando se emplean vínculos débiles antes que fuertes. Ello se debe a las peculiares características de los procesos de difusión de innovaciones.

Un principio obvio de la comunicación humana es que la transmisión de ideas ocurre más frecuentemente entre personas que son parecidas, similares $u$ homogéneas (Rogers, 1983). De hecho, señala Rogers, uno de los problemas más característicos de la comunicación de innovación es que los participantes normalmente son bastante heterogéneos, por ejemplo, el agente de cambio es técnicamente más competente que sus clientes. La naturaleza de la difusión demanda que al menos algún grado de heterogeneidad esté presente entre los participantes. La homogeneidad ocurre frecuentemente en la comunicación porque ésta es más efectiva cuando el emisor y el receptor son homogéneos. La mayoría de los individuos se sienten cómodos interactuando con personas que son bastante similares a ellos. La heterogeneidad, en cambio, puede causar disonancia cognitiva porque un sujeto es expuesto a mensajes que son inconsistentes con sus creencias, causando un estado psicológico incómodo. La homogeneidad y la comunicación efectiva se generan mutuamente: cuanta más comunicación se produce entre dos miembros de una díada, más probable es que se conviertan en homogéneos, y cuanto más homogéneos son, más probable es que su comunicación sea efectiva.

Sin embargo, la comunicación heterogénea tiene un potencial especial informacional, aunque sólo se lleve a cabo raramente. Los lazos heterogéneos de una red a menudo conectan a dos apiñamientos, a dos grupos con individuos socialmente diferentes, y estos lazos interpersonales son especialmente importantes para transmitir información sobre innovaciones. Por ello, la comunicación homogénea puede ser frecuente y sencilla pero no tan crucial como la más infrecuente comunicación heterogénea a la hora de difundir una innovación. 
Cuando se da un cierto grado de heterogeneidad, explica Rogers, existen una serie de características específicas de las personas que lideran el proceso de innovación, los seguidores buscan a los líderes de opinión que tienen un estatus socioeconómico más alto, que tienen más educación, que se exponen más a los medios de comunicación, que son más cosmopolitas, que tienen más contacto con el agente de cambio y que son más innovadores. Estas seis características muestran la tendencia de los seguidores a buscar información y consejo en los líderes de opinión que perciben como técnicamente más competentes que ellos, un fenómeno ya señalado en las investigaciones mencionadas anteriormente. Como se verá en el epígrafe metodológico, las características de los líderes de opinión han servido para crear dos escalas que miden el grado en que un actor es experto en temas comunitarios, una de ellas enfocadas al conocimiento teórico y otra al práctico.

En la investigación llevada a cabo se estudió con quién habla de asuntos europeos más frecuentemente el sujeto, si estas personas que nombra tienen lazos débiles o fuertes con él, medidos a través de la intensidad de la relación y del contenido de ésta. Se quería comprobar si existen personas que realizan la función de puentes locales entre diferentes partes de la red, comprobando si una de estas partes tiene unas relaciones de carácter más localista y otra más cosmopolita. Además, se analizó cómo, a través de estas personas en posiciones de puente y de las conversaciones con otros miembros de su red personal, el individuo forma sus actitudes hacia la Unión Europea y se identifica con ella en mayor o menor medida. ${ }^{6}$

Las reflexiones de Granovetter sobre la importancia de los puentes locales para difundir información fueron empleadas por Burt (2001) para profundizar en este tipo de posiciones. El principal campo de estudio del autor es cómo se crea capital social, especialmente en las organizaciones formales. Frente al argumento de que el capital social es creado por una red de elementos fuertemente interconectados, Burt defiende que se crea a través de una red en que las personas pueden ser intermediarias, es decir, actuar como puentes entre vacíos existentes en la estructura, conectando segmentos que en otro caso estarían aislados. El capital social se crea gracias a que el individuo participa en y controla la difusión de la información. El autor desarrolla su teoría de los vacíos estructurales: los vacíos en la estructura social crean una ventaja competitiva para los individuos cuyas

\footnotetext{
${ }^{6}$ Sin embargo, y como el propio Granovetter advierte (1982), no se puede explicar la adopción de ideas únicamente por medio de consideraciones estructurales, por lo que se hace necesario investigar también el contenido y los motivos para adoptar una idea o símbolo en lugar de otros.
} 
relaciones cruzan estos huecos. Una de las ideas que más interesan en esta investigación de esta teoría - enfocada principalmente al ámbito organizativo y empresarial, ajeno a nuestro objeto de estudio- es que las personas en posiciones de puente, al acceder a mayor cantidad de información y más variada, desempeñan el rol de líderes de opinión porque son los responsables de la difusión de nuevas ideas y comportamientos.

Burt indica que la equivalencia estructural se solapa, restringe y extiende el concepto de cohesión defendido por Coleman. Hay situaciones en que ambos modelos realizan idénticas predicciones: ello ocurre cuando ego y alter tienen estrechas relaciones mutuas $\mathrm{y}$, a la vez, tienen las mismas pautas de relaciones. Tras reanalizar los datos de Coleman, concluye que las predisposiciones personales y la equivalencia estructural explican el proceso de difusión de la nueva medicina. El propio autor considera que tal vez el modelo de cohesión sea una herramienta menos precisa para predecir las percepciones de profesionales altamente cualificados, lo que deja abierta la opción de que la cohesión sea un concepto útil en otros tipos de estructura, como en las redes familiares y de amistades, que es donde se ha desarrollado la mayor parte de la investigación sobre influencia interpersonal y es el tipo de relaciones que interesa en esta investigación. Como se verá en el epígrafe que recoge los resultados, se ha estudiado quiénes ocupan posiciones de mayor intermediación en la red y son puentes entre las distintas partes de ésta. Se supone que dichas posiciones privilegiadas serán ocupadas por individuos que tienen una alta puntuación en la escala de Galtung y que, además, adoptan antes las innovaciones - actitud favorable a la Unión e identidad europeaque el resto de miembros de su red que reciben menos información.

\section{La escala de posición social de Galtung y su uso para estudiar la difusión de nuevas ideas}

Galtung construyó en los años sesenta un índice para situar a las personas en posiciones dentro de la estructura social (Galtung, 1964). Su objetivo al crear esta escala es usar la posición social como variable independiente para explicar las actitudes hacia diferentes asuntos de política internacional. Galtung reconocía que, para que la explicación fuese completa, habría que estudiar también la personalidad del individuo y la estructura de comunicación e influencia de su sistema social, pero defiende el uso de su escala por ser metodológicamente más sencilla de obtener y porque la posición social es la base de la interacción social institucionalizada. 
El autor divide la sociedad en tres partes: un núcleo de decisores (decision-making nucleus), el centro social y la periferia social: dejando de lado a la minoría que toma las decisiones, Galtung define el centro social como aquél que ocupa las posiciones que están socialmente recompensadas, y la periferia social la que ocupa las posiciones menos recompensadas e incluso rechazadas. El autor señala tres diferencias estructurales entre el centro y la periferia: el centro social tiene altos índices de participación social (medidos a través del consumo de medios y su pertenencia a asociaciones) y tiene altos niveles de conocimientos sobre asuntos políticos y de mantenimiento de opiniones sobre esta materia. Frente a ellos, la periferia social tiene una baja participación, pocos conocimientos y no tiene opiniones bien definidas y sólidas.

Galtung considera que, como el centro es quien accede a los medios y además tiene algo que comunicar, el proceso de comunicación se emite desde el centro hacia la periferia. En cuanto a la recepción, aunque gran parte del proceso de comunicación sobre asuntos políticos se da dentro del centro, las personas de la periferia están ligadas a este proceso a través de contactos informales con personas del centro. El autor asume que las nuevas ideas son generadas en el centro social ( $y$, en el caso de que sean importadas, sólo el centro tiene una buena comunicación con el exterior) y posteriormente comunicadas a la periferia.

La escala originaria de Galtung puntúa del 0 al 8 a las personas, otorgando un punto por cada ítem recompensado socialmente que la persona ocupa (centro social) y un 0 si ocupa la posición no recompensada (periferia social). Así, puntúa como 1 ser varón, tener entre 30 y 59 años, tener estudios superiores a los primarios, ingresos por encima de la media nacional, vivir en entorno urbano o suburbano, que su localización geográfica sea central, ser autónomo o trabajador de cuello blanco - frente a los trabajadores manuales, que puntúan 0- y trabajar en los sectores secundario y terciario - frente al primario-. Las personas que puntúan de 0 a 2 se sitúan en la periferia, de 3 a 5 ocupan posiciones intermedias y de 6 a 8 están en el centro social.

Pese a que Galtung elaboró su escala hace más de 40 años, su utilidad ha sido demostrada en numerosas ocasiones. En España, uno de los autores que más han empleado esta escala es Díez Nicolás, que ha actualizado la escala. Así, en la década de los noventa, amplía el tramo de edad recompensado socialmente hasta los 64 años - edad de jubilación-, modificación que también se ha adoptado en esta investigación. Además, debido a la extensión del número de años de educación formal, puntúa 1 el tener estudios posteriores al nivel secundario obligatorio (Díez 
Nicolás, 1992). ${ }^{7}$ Recientemente, el mismo autor ha modificado la escala de nuevo y se han usado varias categorías para cada variable, salvo el sexo, que continúa siendo dicotómico por razones obvias pero modifica sus valores: las mujeres puntúan 1 en vez de 0 y los varones 2 en vez de 1 . De esta manera, el índice se vuelve más rico y se adapta mejor a la compleja sociedad actual, por lo que se han repetido los análisis empleando dichas modificaciones para comparar la capacidad explicativa de ambas escalas. Las variables de este nuevo índice quedan como se explica a continuación (Díez Nicolás, 2005:231):

(i) sexo: mujer $=1$; hombre $=2$; (ii) edad: -18 y $+75=1$; entre 18 y 25 y 65 y 74 años=1; entre 26 y 35 y 55 y $64=2$; entre 36 y 54 años=3; (iii) nivel educativo: menos de estudios primarios y $N S / N C=0$; estudios primarios, $E G B$, bachiller elemental, formación profesional=1; bachillerato superior, BUP, Preu, $C O U=2$; estudios universitarios grado medio y grado superior $=3$; (iv) ingresos: -450 euros=0; 451 a 900 euros $=1 ; 901$ a 1.650=2 y más de 1.650=3; (v) tamaño del hábitat: menos de 10.000 habitantes $=0$; entre 10.000 y 50.000 habitantes $=1$; de 50.000 a 250.000 habitantes $=2$; más de 250.000 y Madrid y Barcelona=3; (vi) estatus ocupacional: no ocupados $+\mathrm{NS} / \mathrm{NC}=0$; no cualificados $=1$; cualificados y medios $=2$; superiores $=3$; ( vii) sector económico: no ocupados $+N S / N C=0$; sector primario/extractivo $=1$; secundario/industria $=2$; terciario/servicios $=3$; ( viii) centralidad: Comunidades Autónomas con renta per cápita baja (Castilla La Mancha, Galicia, Andalucía, Extremadura)=0; Comunidades con renta media (Rioja, Aragón, Cantabria, Comunidad Valenciana, Castilla León, Canarias, Asturias, Murcia)=1; Comunidades con renta per cápita alta (Madrid, Navarra, País Vasco, Baleares, Cataluña) $=2 .^{8}$

\section{Metodología}

\section{Muestreo y generadores de nombres}

En esta investigación se ha empleado el llamado método de muestreo en bola de nieve (snow-ball sampling) para formar la red, por un doble motivo: por un lado, no se estaba interesado en recoger la percepción que el entrevistado tiene de cómo sus amigos, familiares y compañeros valoran la Unión o qué tipo de identidad

\footnotetext{
7 Los ingresos mensuales medios se han actualizado: Díez Nicolás establecía el umbral en 150.000 pesetas y aquí se ha empleado como punto de corte 1.000 euros.

8 Una ventaja de esta nueva escala es que se contempla la posibilidad de que el entrevistado no responda a uno o más ítems o que no esté ocupado.
} 
mantienen, asuntos sobre los que quizá el respondiente no pudiera informar de manera fidedigna - dado que no son tópicos considerados relevantes habitualmentesino que se prefirió preguntar directamente a los mencionados por estas cuestiones; por otro, se quería crear, a partir de las respuestas de los entrevistados, una red más amplia que la propia red personal del encuestado.

El método de la bola de nieve se ha utilizado preferentemente para identificar poblaciones ocultas, mal conocidas o desconocidas. Se trata de un método para obtener una muestra lo más diversa posible y que representa a una población relacionada, aunque desafortunadamente aún se están desarrollando matemáticamente todas sus posibilidades y los análisis que se pueden emplear con ella. ${ }^{9}$ La muestra parte de un sujeto, ego, al que se pregunta por las personas con las que mantiene un tipo específico de relación que interesa al investigador. Todos los mencionados (cada uno es un alter) constituye la llamada zona "de primer orden" de la red. Posteriormente, se entrevista a todos los actores de esta zona y se van incluyendo todas las personas, no mencionadas anteriormente, que forman la zona "de segundo orden", a las que también se entrevista. El proceso sigue a través de diversas zonas, ampliando la red como una bola de nieve que rueda. Con la metodología empleada, la red que se construye no se limita simplemente a una red personal, ya que está formada por múltiples redes personales (una por cada entrevistado que, a su vez, ha mencionado personas con la que mantiene ciertos lazos) y el conjunto de todas ellas forma una red social cuyos límites han sido definidos por el propio investigador. ${ }^{10}$

En este tipo de metodología se plantea el problema de dónde es más adecuado parar, en qué zona se termina la investigación. En este caso, la pregunta fue resuelta con un criterio pragmático: no se estableció a priori cuántas zonas se iban a investigar porque no se sabía cuántos sujetos mencionaría cada respondiente, por lo que se decidió incluir tantas zonas como fueran necesarias para formar una red de una cierta entidad numérica, siempre teniendo en cuenta las limitaciones de presupuesto y tiempo que implica tan costosa metodología para una investigación desarrollada por una sola persona. Finalmente, se entrevistó a los mencionados de las zonas de primer, segundo y tercer orden, zonas que consideramos suficientes

\footnotetext{
9 Para una descripción de la utilización de esta metodología en investigaciones españolas, cfr. Molina (2001).

${ }^{10}$ La delimitación de las fronteras de una red social es, en la mayoría de los casos, problemática. Salvo casos en los que existe una clara frontera que puede ser incluso física - un aula, un hospital-, queda a juicio del investigador definir quiénes forman parte de la red social.
} 
para los fines de la investigación y que han sido empleadas en otras investigaciones. ${ }^{11}$

Se partió de dos egos con características distintas para poder comparar las dos redes y comprobar si los procesos son similares en ambas. Las diferencias entre los dos egos se refieren a edad, nivel educativo y procedencia. Se decidió que ambos egos fueran varones dado que la composición de las redes de varones y mujeres son sensiblemente diferentes y no se quería correr el riesgo de que esta variable distorsionara los resultados. La primera red nace de un ego en el final de su vida activa (cerca de los sesenta años), con formación superior (licenciado) y proveniente de otra Comunidad Autonóma, aunque lleva décadas viviendo en la provincia de Málaga. La segunda red parte de un ego de mediana edad (ronda la cuarentena), con nivel educativo medio (formación profesional) y nacido en la provincia de Málaga, en la que siempre ha vivido. Se planteó como una variable relevante el hecho de cuántas relaciones locales o cosmopolitas tienen los individuos, puesto que se quería comprobar si las personas con un perfil cosmopolita pueden ocupar posiciones de puente entre diferentes partes de la red. Como, a priori, no se podía establecer dicho perfil, se supuso como hipótesis de trabajo que es más probable tener un mayor número de relaciones no locales en aquellas personas que no son originarias del lugar donde residen. ${ }^{12}$

La recogida de información se realizó entre enero y marzo de 2004. El trabajo de campo tuvo lugar en la provincia de Málaga. En el caso de que ego mencionara a personas que viven fuera de esta provincia, se excluyeron del análisis. Cabe decir que esta circunstancia ocurrió en pocas ocasiones $y$, normalmente, estas personas eran mencionadas como miembros de su red más íntima pero con las que no habla con frecuencia de temas políticos - que es el ámbito de interés de esta investigación- precisamente porque la distancia supone pocos contactos y con contenidos más fundamentales y de carácter más privado.

Las entrevistas se realizaron, principalmente, en el domicilio de los encuestados. El hecho de que todas las personas contactadas habían sido avisadas previamente por el ego que las había mencionado para que aceptaran formar parte de la

\footnotetext{
11 Por ejemplo, Katz y Lazarsfeld (1955) utilizaron la técnica de la bola de nieve para estudiar a los líderes de la arena política local de una localidad americana, entrevistando a la muestra original y las tres primeras zonas .

12 Sin embargo, el trabajo de campo dio como resultado que la gran mayoría de los encuestados tenían relaciones localistas y no existían partes de la red de carácter no local, por lo que no se puedo comprobar la hipótesis.
} 
investigación, ayudó a la buena disposición de los entrevistados, que emplearon entre una y dos horas para la realización del cuestionario completo, con una media de alrededor de noventa minutos. Ningún entrevistado puso problemas cuando se solicitó grabar los encuentros. Aunque la mayor parte de las preguntas estaban estructuradas y se debía elegir entre una serie de categorías, se incluyeron algunas preguntas de carácter abierto. La estructura del cuestionario está compuesta de tres bloques, con una primera parte dedicada a la Unión Europea, una segunda a la red personal y una tercera sobre las características sociodemográficas del entrevistado. La segunda parte incluye varios generadores de nombres, los roles que existen entre ego y alter y las características de la relación, así como la conectividad de la red personal del encuestado.

Se han utilizado varios generadores de nombres para crear la red de relaciones más íntimas del sujeto y para diseñar la red de discusión política. El motivo por el que se ha decidido investigar dos tipos de redes se basa en la constatación, por parte de los estudios de sociología política, de que las orientaciones políticas dependen del contexto social del individuo. Por ejemplo, la investigación de Berelson et al (1963) se enfocó hacia el estudio del voto, pero sus conclusiones se pueden ampliar a las actitudes políticas en general. La familia tiene un rol importante en este proceso, hasta el punto de observar una especie de "voto hereditario" ya que la mayoría de las personas continúan la tradición familiar de voto. Por otro lado, los amigos y compañeros de trabajo no son un apiñamiento cerrado, sino que ponen en contacto al sujeto con otras redes de relaciones sociales que afectan su comportamiento político. Estos autores también destacan en su investigación sobre cómo se decide el voto que las amistades actúan como puntos de contacto a través de los que los individuos se conectan con las redes globales de relaciones sociales que afectan a su comportamiento político. Aunque Berelson y sus colegas preguntaron a los encuestados únicamente por sus tres amigos y tres compañeros más íntimos (personas que serían nombradas en el primer generador de nombres), resulta interesante averiguar si esas relaciones son los únicas o existen otras, con las que se mantienen relaciones menos íntimas y normalmente menos frecuentes, pero con las que también se habla de política $y$, por tanto, podrían influir en las actitudes y opiniones del entrevistado. Ello se justifica porque, como Lazarsfeld y Katz habían observado poco antes (1955), los contactos frecuentes no son sinónimo de influencia, hay que tomar en consideración también la valoración que se hace de la competencia y la confianza de los mentores en los asuntos públicos. 
Los tres primeros generadores de nombres forman, conjuntamente, la red de relaciones más íntimas de ego. Se pregunta al entrevistado por las personas con las que hablaría de un problema importante, a las que se pediría consejo antes de tomar una decisión relevante y a las que se pediría dinero. En principio, al sujeto no se le limita el número de nombres que puede decir pero, por motivos de economía, si en conjunto se nombra a más de ocho personas en estas tres preguntas, se pide que limite los mencionados a ocho, circunstancia que ocurrió en pocas ocasiones. ${ }^{13}$ Estos generadores son una adaptación de algunos de los desarrollados por Fischer para el Northern California Community Study (NCCS). ${ }^{14}$ La formulación exacta es la que sigue:

1) Si tuviera un problema personal importante, sea en el ámbito sociolaboral o sea en el afectivo-familiar, ¿con quién hablaría sobre ello? Dígame el nombre y el primer apellido de esa/s persona/s.

2) Si tuviera que tomar una decisión importante, sea en el ámbito sociolaboral o sea en el afectivo-familiar, ¿a quién le pediría consejo? Dígame el nombre y el primer apellido de esa/s persona/s. Una misma persona puede haber sido ya nombrada en la pregunta anterior.

3) Si se viera en un apuro, ¿a quién le pediría prestada una importante suma de dinero? Dígame el nombre y el primer apellido de esa/s persona/s. Una misma persona puede haber sido ya nombrada en las dos preguntas anteriores.

El último generador es original de esta investigación, con él se pretende que el entrevistado nombre a las personas con las que suele hablar con cierta frecuencia de política. En su concepción original, el generador preguntaba con quién se habla de asuntos comunitarios, pero el pre-test mostró que los sujetos contestaban que no hablan de estos temas o sólo de manera tangencial y con poca frecuencia, por lo que se decidió ampliar la pregunta e incluir todos los ámbitos de la política en el entendido de que, en las pocas ocasiones en que la Unión Europea sea el objeto de debate, será con personas con las que se habla habitualmente de otros ámbitos políticos. Tras la modificación, quedó como sigue:

\footnotetext{
${ }^{13}$ Prácticamente, sólo en los casos en que los entrevistados forman parte de una familia con más de seis o siete miembros, ocurría el hecho de que se nombrara a más de ocho personas, y ni siquiera sucedía en todos los casos en que el sujeto tiene este tipo de familia.

${ }^{14}$ Para una descripción detallada de éste y otros generadores, Cf. Requena Santos (1996).
} 
Hablemos ahora de política. Me gustaría que recordara con qué personas habla en alguna ocasión de temas de la actualidad política, bien sea de ámbito local, nacional o internacional. En el caso de la política internacional, puede que en algunas ocasiones se mencionen asuntos europeos (el euro, la política agrícola, los fondos estructurales para construir carreteras, etc.) o de aspectos relacionados con otros países miembros de la UE (lengua, tradiciones, gastronomía...). Es probable que algunas de estas personas con las que habla de política hayan sido mencionadas anteriormente, pero otras puede que sean conocidos con los que el contacto sea menos frecuente o menos íntimo. Me gustaría que me dijera si alguna de esas personas fue mencionada anteriormente y si existe alguna persona más que aún no ha sido nombrada. Si es así, dígame el nombre y su primer apellido.

Como en el caso de la red más íntima, en principio no se limitó el número de personas que el entrevistado podía mencionar pero, si enumeraba más de cinco personas nuevas (no nombradas anteriormente con los otros tres generadores) se pedía que restringiera a cinco los nombrados. Por tanto, trece personas son el máximo que podía mencionar ego entre todos los generadores. A continuación se pedía al entrevistado que señalara todas las posibles relaciones que le unían con cada uno de los mencionados. Las relaciones con las personas nombradas en los tres primeros generadores fueron clasificadas como "íntimas" de ego, mientras que, para el cuarto generador, se pidió al sujeto que calificara su grado de intimidad con cada mencionado. Para todas las relaciones se preguntó hace cuánto tiempo que se conocen, con qué frecuencia se ven en persona, con qué frecuencia mantienen contacto no personal (correo, teléfono, etc.) y a qué distancia viven. Por último, se pidió al individuo que indicara si cada par de nombres se conoce entre sí y qué grado de intimidad tienen.

\section{Creación de las escalas de expertos}

Como se señaló en el primer epígrafe, las conclusiones sobre cuáles son las características de las personas que ejercen un liderazgo de opinión en su grupo llevó a crear dos escalas para comprobar la hipótesis de que son las personas más familiarizadas con la Unión y más informadas sobre ella las que ejercen más influencia en la red y ocupan posiciones más centrales en ella. Además, y basándonos en el enfoque del cosmopolitismo desarrollado por Inglehart (1977, 1990) y Deutsch (1952), se suponía que estos expertos son más favorables al proyecto europeo y quienes se identifican más a menudo como europeos, aunque los resultados no se muestran aquí por falta de espacio.

Se crearon a posteriori las escalas de "experto teórico" y "experto práctico": los primeros basan su conocimiento sobre la UE en su mayor participación política, mayor nivel de conocimientos de las políticas comunitarias, mayor consumo de 
medios, etc., mientras que los segundos están más familiarizados con la UE porque mantienen lazos con personas de otros países comunitarios, viajan con cierta frecuencia a estos países y permanecen más tiempo en ellos. Las personas no tienen por qué puntuar necesariamente alto en ambos tipos de escalas $y$, de hecho, los resultados mostraron que la correlación es bastante baja, de 0,215* (Pearson).

Tras diversas pruebas con diferentes variables para maximizar la varianza explicada con el análisis factorial, la escala de experto teórico incluye siete variables construidas a partir de varias preguntas del cuestionario: (i) Grado de conocimiento de los derechos de la ciudadanía europea; (ii) grado de conocimiento de las instituciones europeas; (iii) grado de participación social: suma de la participación en organizaciones de distinto carácter; (iv) consumo total de medios de comunicación; (v) grado de interés por la política; (vi) frecuencia con la que se habla de asuntos políticos y (vii) frecuencia con la que se intenta persuadir de una opinión política. ${ }^{15}$

La escala de experto práctico se creó con tres ítems: número total de viajes a otros países de la UE, periodo más largo de estancia en uno de estos países y relación más estrecha que tiene con personas de estos países. Los dos primeros ítems fueron propuestos por Deutsch (1952) como variable estructurales relevantes para desarrollar una visión cosmopolita, más favorable por tanto a una identidad europea, mientras que el tercero es puramente reticular.

\section{Resultados}

\section{Características de las redes de discusión política}

Los 108 entrevistados ( 52 en la red primera y 56 en la red segunda) nombraron 800 relaciones, aunque las relaciones entre todos los entrevistados (los dos egos más primer, segundo y tercer círculo) son 332. A continuación se comentan algunas características de estas relaciones prestando especial atención a la red de discusión política. Los porcentajes de relaciones que no son nombradas en el generador de discusión política son bastante similares en las dos redes: en la primera, son un 12,6\% (22 de 175) y en la segunda un 11,5\% (18 de 157). Aunque el cuestionario incluye una detallada tabla de todos los tipos de relaciones

\footnotetext{
15 Se decidió no incluir la variable "estudios" por dos motivos: por un lado, disminuía el grado de varianza explicada, por otro, el nivel de estudios se ha mostrado en numerosos estudios como uno de los factores más relevantes para analizar las actitudes de un sujeto y, al ser incluída en una escala con más ítems, se diluye su capacidad explicativa.
} 
que se pueden tener, por motivos de claridad expositiva se han agrupado en cinco: roles familiares, de amistad, de compañeros de trabajo y/o estudios, de vecindad y otro tipo de relaciones (participación en actividades organizadas, asociaciones, partidos, sindicatos, etc.). En teoría, el valor máximo que se puede encontrar es 5 (ego mantiene un rol con alter en cada uno de estos ámbitos) aunque en las dos redes lo máximo que se ha hallado es el valor 4.

Se pidió a los entrevistados que definieran la intensidad de la relación con cada uno de los mencionados en la red política. Las posibilidades que se ofrecían eran: valor 1 para las personas que se conocen superficialmente, valor 2 para las personas consideradas "amigas" y valor tres para los "amigos íntimos". En ambas redes, el porcentaje de personas con las que se habla de política sin ser íntimos es alrededor del $20 \%$, aunque es algo más frecuente en la red segunda $(20,2 \%)$ que en la primera $(18,4 \%) .{ }^{16}$ Este dato es relevante porque muestra que la mayor parte de la información sobre asuntos políticos se comparte entre personas que tienen lazos fuertes, pero existe un grupo de relaciones que, si bien son minoritarias, pueden ser fundamentales - tal y como apunta Granovetter- para difundir información novedosa por tratarse de lazos débiles que tienen contacto exterior con otras personas.

Para profundizar en quiénes son estas personas con lazos débiles, se ha comprobado qué roles tienen ego y alter en estos casos. En la red primera, el rol que tiene más amigos no íntimos es el vecinal (un 44\%), al que le sigue, precisamente, el rol de amistad (33\%); en cambio, son mucho más minoritarios los amigos no íntimos entre los compañeros de trabajo y/o estudio (13\%) y las personas con las que se mantienen otro tipo de relaciones $(8 \%)$. Como dato curioso, se puede comentar que el $3 \%$ de las relaciones familiares de esta red se califican como de no íntimas, algo que no ocurre en ningún caso en la segunda red. Precisamente, en la red segunda el rol más común entre las personas que no son íntimos es el de amistad (30\%), seguido de otro tipo de relaciones (25\%) y el rol vecinal (20\%), mientras que el caso más minoritario es el de los compañeros de trabajo/estudios (17\%), aunque la cifra es superior a la encontrada en la primera red. Por tanto, aunque la distribución es diferente en ambas redes, parece común a ambas que la intimidad es mayor, aparte de en las relaciones familiares, en las de los compañeros de trabajo/estudios, mientras que alrededor de un tercio de las

\footnotetext{
${ }^{16}$ Sólo una se ha definido como "conocido superficialmente" y se trata de una relación vecinal, el resto de mencionados son considerados amigos aunque sin llegar a ser íntimos.
} 
personas con las que se mantiene un rol de amistad se califica de no íntimos. La diferente distribución de los no íntimos en las otras relaciones tiene, probablemente, su explicación en que en la primera red fueron clasificadas en este rol las personas con las que se comparte la pertenencia a instituciones religiosas, en las que se fomenta precisamente la hermandad y estrechamiento de lazos, mientras que en la segunda se comparte la afiliación a partidos políticos, en los que la intimidad entre los participantes no es tan necesaria, requerida ni valorada como en el caso anterior. Por otro lado, los vecinos de la red segunda se consideran mucho más íntimos que los de la red primera, pero en este caso hay que tener en cuenta que en la segunda ocurre con más frecuencia que los vecinos sean además familiares. Por tanto, volviendo a la propuesta de Granovetter, parece que la forma más probable -a través de lazos débiles- de que llegue nueva información a una persona es mediante sus relaciones de amistad con personas no íntimas, mientras que resulta más improbable a través de la familia y de los compañeros de trabajo/estudios.

En la red primera es más frecuente hablar de política cuanto más contenidos (recogido cada uno de ellos en un generador de nombres) existan entre ambos actores, en la red segunda ocurre al contrario. En la red primera se habla más de política con personas a las que se les cuenta un problema que en la segunda ( $56 \%$ frente a $40 \%$ ) y se les pide dinero ( $26 \%$ frente a $16 \%$ ), mientras que tiene porcentajes similares en las dos redes la frecuencia con que se habla de ella con personas a las que se les pide consejo (alrededor del 45\%). En la red primera, cuantos más roles se establecen entre ego y alter -cuanto más multiplicidad de roles exista- más probable es que ambos hablen de política, en la red segunda la tendencia no es lineal, ya que las personas con dos roles hablan más que las que tienen rol único, pero las de tres roles hablan menos que las de dos. En todo caso, el número de personas con dos o tres roles en la segunda red es alrededor de la mitad que en la red primera. En ambas redes todas las personas que tienen cuatro roles habla de política con ego, aunque son muy pocas, tres en total.

Los miembros de la red primera hablan menos de política con los amigos que los de la segunda (dos puntos porcentuales de diferencia) y con las personas con las que tienen otro tipo de relaciones, pero hablan más con los familiares (tres puntos) y compañeros de trabajo/estudio. En las dos redes se habla con los vecinos de este asunto, excepto un caso en la red segunda, aunque el número de estas relaciones es la mitad en la red primera que en la segunda. Las dos mayores diferencias (siete puntos en ambos casos) entre las dos redes se encuentran en las personas con las 
que existen otro tipo de relaciones (miembros de asociaciones, etc.) y en los compañeros de trabajo/estudio: en la red segunda se habla mucho más con los primeros de política - y el número de estas relaciones duplica al de la otra redmientras que en la red primera es con los colegas de trabajo o estudio.

El primer fenómeno se puede explicar porque parte de los miembros de la red son militantes de un partido político y se reúnen semanalmente para, precisamente, hablar de asuntos de la arena política. En la red primera, en cambio, la mayoría de las "otras relaciones" se refieren a miembros de agrupaciones religiosas, cuyo motivo principal de reunión no es la discusión política. En cuanto al segundo fenómeno, en la red primera gran parte de los mencionados que fueron entrevistados formaban parte del ámbito laboral de ego, de hecho el número de este tipo de relaciones en la red primera quintuplica a la de la red dos. Todos estos compañeros mantienen otro tipo de relación adicional con ego, sea familiar, de amistad u otra, y por ello es más probable que se produzca una discusión política, no necesariamente en el contexto laboral, que si únicamente se compartiera el tiempo y espacio de trabajo.

\section{Medidas estructurales de las redes y de los actores}

En este apartado se van a analizar algunas medidas estructurales que caracterizan a las redes en su conjunto y a sus miembros individualmente, para relacionarlas posteriormente con las escalas de experto práctico y teórico. La tabla 1 recoge, de manera separada para cada red, la densidad (el porcentaje de todos los posibles lazos que están presentes en una red, medida inversamente relacionada con el tamaño de la red), la centralización de la red tanto total como indegree y outdegree (que mide cuánto varían los vértices con respecto a su rango total, de entrada y de salida, cuyos valores varían entre 0 y 1 ) y el grado de intermediación (que se refiere a cuánto difieren los nodos en función de su intermediación, es decir, un nodo es intermediario cuando está en la geodésica entre dos puntos). ${ }^{17}$

\footnotetext{
17 Todas las definiciones empleadas han sido extraídas de De Nov y Batagelj (2004). No se ha podido emplear la medida de cercanía (closeness) porque hay actores aislados en la red.
} 


\begin{tabular}{|c|c|c|}
\hline & Red primera & Red segunda \\
\hline Centralización total & 0,08902 & 0,10438 \\
\hline Centralización indegree & 0,18185 & 0,13950 \\
\hline Centralización outdegree & 0,10188 & 0,12099 \\
\hline Densidad $^{18}$ & 0,0569382 & 0,0448052 \\
\hline Grado de intermediación & 0,17676 & 0,28981 \\
\hline
\end{tabular}

Tabla 1. Propiedades estructurales de las redes

Observando la tabla 1 se comprueba que la red segunda está más centralizada si se tiene en cuenta el rango total (es decir, la suma de los rangos de entrada y salida), y el rango de salida, (outdegree) aunque las diferencias con la red primera no son muy altos, y en ambos casos estas cifras son pequeñas, de no más del $14 \%$. La mayor centralización se produce en el rango de entrada (indegree) en la red primera, que sobrepasa el $18 \%$. La red primera es más densa que la segunda, hecho en parte esperable dado que es más pequeña. Por último, existe una mayor concentración de los valores de intermediación en la red segunda, una diferencia bastante sensible ( $28 \%$ frente a $17 \%$ ). Este hecho se ve corroborado en la tabla 2 , en la que se muestra que la persona con mayor intermediaciónen la red segunda tiene una puntuación mucho más alta que la de su equivalente de la red primera.

La tabla 2 muestra las medidas reticulares de los actores tomados que forman cada una de las redes. Además de las medidas comentadas interiormente, se incluye el dominio de entrada (input domain) total y restringido y el prestigio de proximidad (proximity prestige). En las redes dirigidas, se considera que son prestigiosas las personas que reciben muchas elecciones positivas. El prestigio se convierte en especialmente importante si las elecciones positivas no son recíprocas, en estos casos el prestigio social está conectado con el poder social. El prestigio estructural no es idéntico al concepto de prestigio social que se utiliza normalmente en las ciencias sociales, aunque probablemente esté relacionado con él (De Nov y Batagelj, 2004). El rango de entrada o indegree de un vértice es el número de flechas que recibe en una red dirigida. Es la medida más simple de prestigio estructural pero sólo se puede emplear en redes dirigidas. Es una medida restringida porque toma en cuenta únicamente las elecciones directas, no importa si quien elige es gente que a su vez es elegida por muchos o que no es elegida por 
nadie, por lo que la estructura entera de la red no se toma en consideración. Para tener en cuenta las elecciones indirectas, se han utilizado medidas como el dominio input - de entrada- de un actor, que es el número o porcentaje de otros actores que están conectados por un camino con él. En una red muy bien conectada con muchas relaciones recíprocas, los vértices son alcanzables por la mayoría de los otros vértices, por lo que las puntuaciones del dominio varían poco. En estos casos es más interesante para capturar la estructura de la red usar un índice restringido, por ejemplo las personas que son alcanzables en uno o dos pasos - directamente y a través de un único intermediario-, empleado en esta investigación.

Sin embargo, la elección de una distancia máxima dentro de un dominio de entrada restringido es arbitraria, problema que se supera con el concepto de prestigio de proximidad (proximity prestige). Este índice de prestigio considera todos los vértices dentro del dominio input de un vértice pero les concede más importancia a las nominaciones si son expresadas por un vecino próximo: es decir, las nominaciones de personas próximas contribuyen más al prestigio de proximidad de un actor que la nominación por parte de alguien distante, aunque varios distantes contribuyen tanto como uno cercano. Lo que hace esta medida es ponderar cada elección en función de la distancia que hay hasta el vértice. El cálculo se realiza dividiendo el dominio input de un vértice por la distancia media de todos los vértices en su dominio. Todas estas medidas basadas en las elecciones que recibe un actor están, obviamente, altamente relacionadas entre sí.

\begin{tabular}{|l|c|c|}
\hline \multicolumn{1}{|c|}{ Variables reticulares } & Red primera & Red segunda \\
\hline Valor más alto normalizado de todos los grados & $0,1274510(13)$ & $0,1454545(16)$ \\
\hline Valor más alto normalizado de rango de entrada & $0,2352941(12)$ & $0,1818182(10)$ \\
\hline Valor más alto normalizado de rango de salida & $0,1568627(8)$ & $0,1636364(9)$ \\
\hline Valor más alto de intermediación & 0,2010784 & 0,3117284 \\
\hline Valor más alto de prestigio de proximidad & 0,3313968 & 0,2797203 \\
\hline Valor más alto normalizado de dominio de entrada & $0,82352941(42)$ & 0,78181818 (43) \\
\hline $\begin{array}{l}\text { Valor más alto normalizado de dominio de entrada } \\
\text { restringido }\end{array}$ & $0,41176471(23)$ & $0,34545455(19)$ \\
\hline
\end{tabular}

Tabla 2. Medidas reticulares de los actores de la red ${ }^{19}$

\footnotetext{
${ }^{18}$ La medida de densidad que se incluye aquí no permite "loops", es decir, autoelecciones, ya que no tienen sentido en una red de discusión política.

${ }^{19}$ Entre paréntesis se encuentran los números absolutos (número de lazos).
} 
Esta tabla muestra que la persona que tiene un mayor número de nominaciones por parte del resto de los miembros de la red, tanto en números absolutos como en proporción al total de posibles lazos de entrada, forma parte de la red primera, mientras que la red segunda tiene a la persona con el valor más alto de rango de salida y con el valor más alto total, sumando salida y entrada. La persona de la red segunda con mayor intermediación puntúa sensiblemente por encima de su homónimo de la red primera (0,31 frente a 0,20, un 50\% más). La medida de prestigio de proximidad, en cambio, es mayor en la red primera, así como el dominio de entrada, tanto total como restringido. La persona con un mayor dominio de entrada en la red primera está conectada al $82 \%$ de la red y la de la red segunda al $78 \%$. Si sólo se tienen en cuenta las elecciones directas y las que se tienen a través de un intermediario, el sujeto que puntúa más alto en la red primera se relaciona con el $41 \%$ de la red, y el de la segunda con el $34 \%$. Este hecho es esperable dado que la primera red es más densa que la segunda y, por tanto, todos sus miembros están mejor conectados y les resulta más fácil entrar en contacto entre sí, teniendo unos dominios de entrada mayores que los de la red segunda, menos densa. El índice de prestigio de proximidad, por otra parte, señala que en la red primera de discusión política hay personas con un prestigio mayor que en la segunda.

Se ha querido comprobar cuál es la relación entre estas medidas estructurales y las escalas de experto práctico y teórico que se crearon a partir de varias preguntas del cuestionario. Las escalas se crearon a posteriori, una vez recogida la información, pero se basan en la hipótesis de que las variables que se incluyen en ellas hacen percibir al sujeto que puntúa alto en ellas como alguien "que sabe", alguien "experto" y que, por tanto, ocupa una posición relevante en la red de discusión política. Ya se ha visto que medidas como cuántas personas mencionan al individuo, a cuántas puede acceder de forma indirecta o sólo en dos pasos, si ocupa posiciones importantes de intermediario o su prestigio de proximidad son buenos indicadores de su centralidad y relevancia. Es de esperar, por tanto, que exista una relación entre la puntuación en dichas variables reticulares y la puntuación en las escalas de experto teórico y práctico.

Además, se analiza también la asociación con el rango de salida (outdegree), es decir, el número de flechas que envía en una red dirigida. Cabe suponer que los expertos - al menos los teóricos, teniendo en cuenta las variables que se incluyen en su escala- son miembros activos de la sociedad e interesados por la política, por lo que probablemente mencionan a muchas personas con las que hablan de 
asuntos públicos. Por último, se ha tenido en cuenta que las probabilidades de ser mencionado por otros actores son distintas según a qué estrella pertenece el actor - es decir, en qué momento de la bola de nieve fue mencionado-: por ejemplo, una persona de la estrella de primer orden tiene probabilidad de ser mencionada además de haber sido nombrada en primer lugar por ego- por otros actores tanto de primer orden como de segundo y tercer orden, mientras que una que forma parte de la estrella de tercer orden -además de haber sido mencionada por al menos una persona de segundo orden- ya sólo puede ser mencionada por personas de su mismo orden. Por ello, se han querido incluir también las medidas de popularidad teniendo únicamente en cuenta por cuántas personas del mismo orden es mencionado un mismo actor, así como a cuántas personas de la misma estrella menciona dicho sujeto.

Los datos muestran que la hipótesis se confirma en el caso de los expertos teóricos pero no en el de los prácticos, ya que no existe ninguna correlación significativa entre la escala de experto práctico y las medidas reticulares. Parece, por tanto, que la experiencia internacional, a través de viajes y relaciones con otros países y ciudadanos de la UE, no se perciben como relevantes por el resto de miembros de la red para considerar que alguien es un experto, pero sí factores como el nivel de conocimientos de las instituciones comunitarias y de la ciudadanía europea, la participación en asociaciones, el interés por la política, etc. Excepto el rango de salida, todas las demás variables estructurales se relacionan positiva y significativamente con la escala de experto teórico. ${ }^{20}$ Las variables más relevantes son el prestigio de proximidad $\left(0,462^{* *}\right)$ y, a mayor distancia, el dominio de entrada restringido y dominio de entrada total $(0,398 * *$ y $0,384 * *$, respectivamente). Cabe destacar que las variables que tienen en cuenta los lazos de entrada y de salida para cada estrella por separado son significativas, aunque con una significación menor en el caso del rango de salida. Por último, también tiene una menor significación el grado de intermediación. En conclusión, las personas que puntúan alto en esta escala suelen ser muy mencionadas por el resto de los miembros de la red de forma directa o indirecta y tienen un prestigio estructural dentro de ésta.

\footnotetext{
${ }^{20}$ Se han comprobado estas correlaciones para cada red por separado y los resultados son similares a las dos redes conjuntas, salvo que en la red segunda no existe correlación significativa entre la intermediación y la escala de experto teórico. Las correlaciones son más fuertes en todos los casos en la red primera que en la segunda.
} 


\begin{tabular}{|l|c|c|}
\hline & $\begin{array}{c}\text { Escala de experto } \\
\text { teórico }\end{array}$ & $\begin{array}{c}\text { Escala de experto } \\
\text { práctico }\end{array}$ \\
\hline Rango de entrada & $0,355^{* *}$ & 0,022 \\
\hline Rango de entrada por estrellas & $0,285^{* *}$ & $-0,103$ \\
\hline Intermediación & $0,238^{*}$ & 0,002 \\
\hline Dominio de entrada & $0,384^{* *}$ & 0,111 \\
\hline Dominio de entrada restringido & $0,398^{* *}$ & 0,059 \\
\hline Prestigio de proximidad & $0,462^{* *}$ & 0,076 \\
\hline Rango de salida & 0,160 & 0,050 \\
\hline Rango de salida por estrellas & $0,214 *$ & $-0,042$ \\
\hline
\end{tabular}

Tabla 3. Correlaciones entre las escalas de experto y medidas reticulares Correlaciones de Pearson. Significación: * $\mathrm{p}<0,05 * * \mathrm{p}<0,01$

El siguiente análisis pretende confirmar la hipótesis de que las personas que puntúan alto en las escalas de experto teórico y práctico ocupan posiciones centrales en la red. ${ }^{21}$ Para comprobar la hipótesis se han tomado dos variables reticulares que indican la centralidad de una persona: el grado de intermediación y el prestigio estructural. Ambas variables han mostrado correlaciones positivas significativas con la escala de experto teórico -recogidas en la tabla 3- pero, en este caso, no se trata de ver qué asociación general existe entre las puntuaciones de todos los entrevistados en estas variables y sus puntuaciones en las escalas, sino únicamente tomar en consideración las diez personas con mayor intermediación y con mayor prestigio estructural en cada red. Por último, como se supone que son las personas más centrales en la estructura social las que ocupan posiciones relevantes de intermediación y de prestigio de proximidad, se ha incluido cuál es la posición social de Galtung - tanto la primera versión de la escala como la segunda, explicadas anteriormente- para cada una de las diez personas estudiadas en cada red. Los resultados se muestran en las tablas 4 y 5 .

\footnotetext{
${ }^{21}$ También se comprobó que las personas que ocupan posiciones centrales en la red tienen una actitud más positiva hacia la Unión que el resto, hipótesis que quedó confirmada aunque no se muestran aquí los resultados por falta de espacio.
} 


\begin{tabular}{|c|c|c|c|c|c|c|c|c|}
\hline \multirow{2}{*}{$\begin{array}{c}\text { Grado de } \\
\text { intermediación } \\
\text { de ego }\end{array}$} & \multicolumn{4}{|c|}{ Red primera } & \multicolumn{4}{|c|}{ Red segunda } \\
\hline & $\begin{array}{c}\text { Escala } \\
\text { Galtung } \\
1\end{array}$ & $\begin{array}{c}\text { Escala } \\
\text { Galtung } \\
2\end{array}$ & $\begin{array}{l}\text { Experto } \\
\text { teórico }\end{array}$ & $\begin{array}{l}\text { Experto } \\
\text { práctico }\end{array}$ & $\begin{array}{c}\text { Escala } \\
\text { Galtung } \\
1\end{array}$ & $\begin{array}{c}\text { Escala } \\
\text { Galtung } \\
2\end{array}$ & $\begin{array}{l}\text { Experto } \\
\text { teórico }\end{array}$ & $\begin{array}{l}\text { Experto } \\
\text { práctico }\end{array}$ \\
\hline 1 & 7 & 17 & 4 & 4 & 7 & 17 & 2 & 3 \\
\hline 4 & 8 & 17 & 4 & 4 & Jubilado & 13 & 4 & 3 \\
\hline 5 & 8 & 18 & 4 & 1 & Jubilada & 10 & 4 & 3 \\
\hline 6 & 7 & 17 & 3 & 3 & 8 & 18 & 3 & 1 \\
\hline 7 & Jubilada & 10 & 4 & 1 & Jubilado & 11 & 3 & 4 \\
\hline 8 & 8 & 19 & 1 & 2 & 7 & 18 & 4 & 3 \\
\hline 9 & 8 & 18 & 2 & 2 & $\begin{array}{c}\text { Ama de } \\
\text { casa }\end{array}$ & 8 & 4 & 2 \\
\hline
\end{tabular}

Tabla 4. Relaciones entre el grado de intermediación, la posición social de Galtung y las escalas de expertos y la actitud hacia la UE (en cuartiles)

La tabla 4 incluye las diez personas con mayor intermediación en cada una de las redes, la posición que ocupan en la escala de Galtung y en qué cuartil se sitúan en las escalas de experto teórico y experto práctico. Con el fin de aumentar la claridad expositiva, se incluyen las puntuaciones que obtienen en la medida de intermediación se recogen, junto al resto de los miembros de su red, en las representaciones de las dos redes que se incluyen en las figuras 1 y 2 .

Hay que recordar que sólo puntúan en la primera versión del índice de Galtung las personas empleadas. De los 108 entrevistados, 25 son inactivos y por ello no se ha calculado su puntuación en esta escala. La mayoría de las posiciones de Galtung de las dos redes son altas, pues el $80 \%$ de los entrevistados ocupan posiciones 7 y 8 , entre otros motivos porque, salvo una excepción, todos viven en ámbitos urbanos y la mayoría en la capital. Por ello no sorprende que las personas con mayor intermediación puntúen precisamente 7 y 8 . En cuanto a la segunda versión de la escala, teniendo en cuenta que todos los encuestados puntúan 0 en la variable "centralidad" porque residen en Andalucía, la puntuación máxima que pueden obtener es 20 puntos. Las puntuaciones más numerosas en las dos redes tomadas conjuntamente son 17 y 18 ( 23 casos cada una). En este caso los inactivos sí obtienen una puntuación, aunque ésta no puede ser muy alta al puntuar 0 en los dos ítems relacionados con el empleo. Los inactivos que ocupan posiciones relevantes de intermediación son en su mayoría jubilados jóvenes (no superando los 65 años). En el caso de las mujeres de la red 1, han sido nombradas sobre todo por sus familiares, mientras que en la red 2 los jubilados varones son mencionados sobre todo por amigos, y la mujer ama de casa por amigos y familiares. Todos los jubilados -si estuvieran aún activos- formarían parte también, por ocupación, ingresos, estudios, etc., del tramo más alto de ambas escalas de Galtung. El ama de casa, en cambio, es muy mencionada entre sus amigos porque es militante de 
una agrupación local de un partido político y por ello es muy conocida en su distrito pero ocuparía posiciones intermedias aunque por debajo de la mitad en la segunda versión del índice de Galtung. En la red primera, nadie puntúa por debajo de 10 (la mitad de la puntuación posible) y sólo dos tienen esta puntuación media, el resto se sitúa en 17, 18 y 19 puntos. En la segunda sólo hay una persona por debajo de 10 (el ama de casa), una tiene diez puntos y el resto está por encima de la mitad, aunque en posiciones en general menos centrales que en la primera red.

Se ha comprobado la hipótesis de que las personas con puntuaciones altas en las escalas de experto práctico y teórico ocupan posiciones de intermediación relevantes en la red. Para ello, se han dividido las puntuaciones para ambas escalas, en cada red por separado, en cuatro cuartiles, comprobando a qué cuartil pertenece, de menor puntuación (1) a mayor (4) las diez personas con mayor intermediación de cada red. En el caso de los expertos teóricos, la hipótesis se confirma porque sólo hay un $20 \%$ en los dos cuartiles inferiores y un $80 \%$ en los dos superiores, para ambas redes. En el caso de los expertos prácticos, se cumple en la red segunda y no en la primera, pero en ambos casos los dos tramos (los dos superiores y los dos inferiores) están mucho más igualados: en la red segunda el $60 \%$ pertenecen a los cuartiles 3 y 4 , en la red uno solamente el $40 \%$.

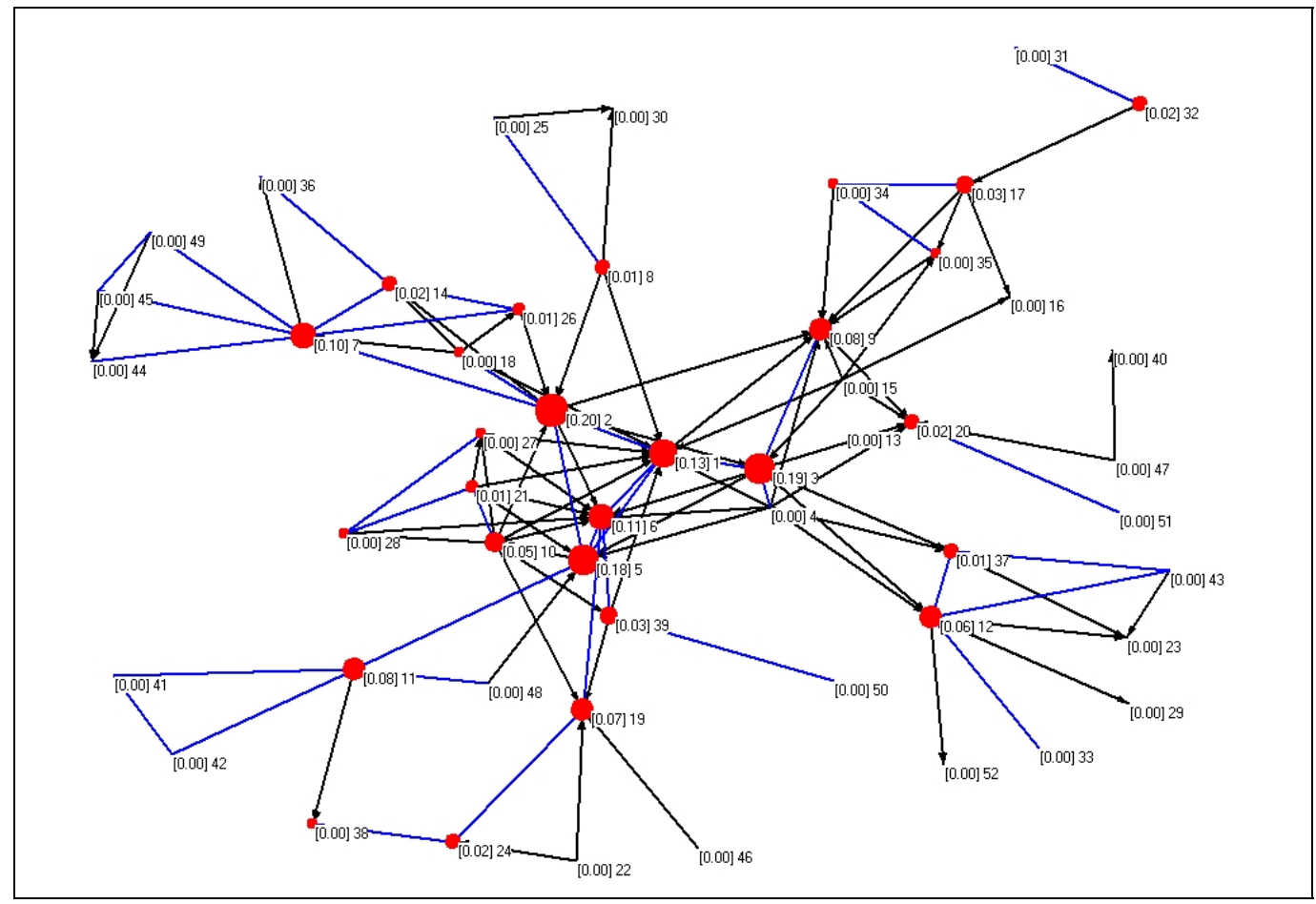

Figura 1. Valores de intermediación de la red primera 


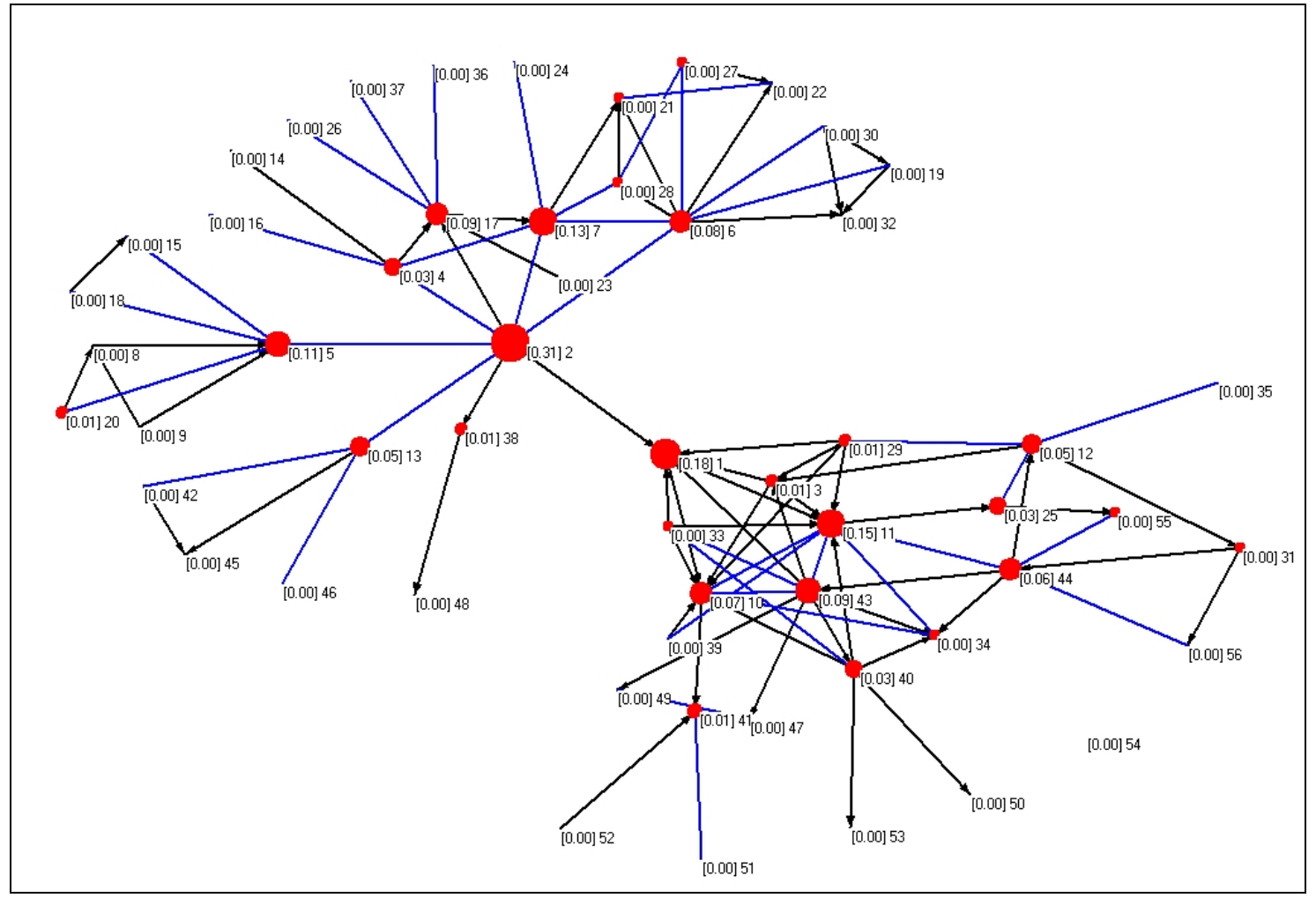

Figura 2. Valores de intermediación de la red segunda

El mismo método se ha empleado para ver la relación entre el prestigio de proximidad y las escalas de experto. Hay que aclarar que los resultados son muy similares a los anteriores porque, en gran parte, coinciden los individuos que tienen una mayor intermediación con los que tienen un mayor prestigio de proximidad (ocho coincidencias en la red primera y siete en la segunda).

\begin{tabular}{|c|c|c|c|c|c|c|c|c|}
\hline \multirow{2}{*}{$\begin{array}{c}\text { Grado de } \\
\text { prestigio } \\
\text { proxim. de ego }\end{array}$} & \multicolumn{4}{|c|}{ Red primera } & \multicolumn{4}{|c|}{ Red segunda } \\
\hline & $\begin{array}{c}\text { Escala } \\
\text { Galtung } \\
1\end{array}$ & $\begin{array}{c}\text { Escala } \\
\text { Galtung } \\
2\end{array}$ & $\begin{array}{l}\text { Experto } \\
\text { teórico }\end{array}$ & $\begin{array}{l}\text { Experto } \\
\text { práctico }\end{array}$ & $\begin{array}{c}\text { Escala } \\
\text { Galtung } \\
1\end{array}$ & $\begin{array}{c}\text { Escala } \\
\text { Galtung } \\
2\end{array}$ & $\begin{array}{l}\text { Experto } \\
\text { teórico }\end{array}$ & $\begin{array}{l}\text { Experto } \\
\text { práctico }\end{array}$ \\
\hline 1 & 8 & 18 & 3 & 2 & 7 & 15 & 2 & 1 \\
\hline 2 & 8 & 17 & 3 & 4 & 7 & 16 & 4 & 1 \\
\hline 3 & 8 & 18 & 4 & 2 & $\begin{array}{c}\text { Ama de } \\
\text { casa }\end{array}$ & 8 & 4 & 2 \\
\hline 4 & Jubilada & 10 & 3 & 1 & 7 & 17 & 2 & 3 \\
\hline 5 & 7 & 17 & 4 & 4 & 8 & 18 & 3 & 4 \\
\hline 6 & Jubilada & 10 & 3 & 2 & Jubilado & 13 & 4 & 3 \\
\hline 7 & 8 & 18 & 2 & 2 & 8 & 18 & 4 & 4 \\
\hline 8 & 8 & 20 & 4 & 4 & 7 & 16 & 1 & 2 \\
\hline 9 & 7 & 17 & 2 & 4 & 8 & 18 & 3 & 1 \\
\hline 10 & 8 & 19 & 2 & 2 & Jubilada & 9 & 1 & 4 \\
\hline
\end{tabular}

Tabla 5. Relaciones entre el prestigio de proximidad, la posición social de Galtung, las escalas de expertos y la actitud hacia la UE (en cuartiles) 
En la red primera, no hay nadie en el cuartil 1, hay tres en el 2, cuatro en el 3 y tres en el 4: se confirma la hipótesis porque sólo el $30 \%$ está por debajo de la media. En la escala de experto práctico, por su parte, hay uno en el primero, cinco en el segundo, nadie en el tercero y cuatro en el cuarto: en este caso no se confirma la hipótesis, puesto que hay más gente por debajo que por encima de la media ( $60 \%$ frente a $40 \%$, aunque ese $40 \%$ se sitúa únicamente en el cuartil más alto de la escala, mientras que en el $60 \%$ sólo un caso está en el cuartil más bajo). En la red segunda, en la escala de experto teórico hay dos personas en el cuartil primero, dos en el segundo, dos en el tercero y cuatro en el cuarto: por lo tanto, un $60 \%$ está por encima de la media y existe una relación entre tener un prestigio de proximidad y ser un experto teórico. En el experto práctico hay tres individuos situados en el cuartil primero, dos en el segundo, dos en el tercero y cuatro en el cuarto. En esta ocasión, a diferencia de las puntuaciones sobre la intermediación, no se confirma tampoco en esta red la hipótesis, pues las personas se reparten a partes iguales en los dos primeros cuartiles y los dos últimos. En cuanto a la escala de posición social, de nuevo no hay nadie por debajo de 10 en la red primera y dos con esa puntuación, el resto puntúa 17 o más e incluso se produce el caso de un actor con la máxima puntuación posible (20). En la red segunda hay dos personas por debajo de 10 y el resto ofrece una distribución parecida a la tabla 4.

\section{Conclusiones}

A lo largo de este artículo, se han mostrado los resultados de una investigación en que se quería estudiar el papel ejercido por los expertos en un determinado asunto (en este caso, la UE) en la red de la que forman parte. La hipótesis principal que se quería contrastar es que los expertos son percibidos como tales por el resto de los miembros y por ello ocupan posiciones centrales en las redes. El trabajo de campo se produjo en un único momento en el tiempo por lo que no se podía realizar un estudio de la difusión de las actitudes favorables a la Unión y la identidad europea en un cierto periodo pero, adoptando la hipótesis de Galtung de que las nuevas ideas parten del centro social hacia la periferia, se ha comprobado si dichos expertos ocupan posiciones recompensadas socialmente en la estructura social. Por último, se quiso comprobar si, como implica la teoría de la fuerza de los lazos débiles de Granovetter, estos vínculos son relevantes en las redes de discusión política. Como resumen de los resultados mostrados, se puede concluir: 
1) La hipótesis de que los expertos ocupan posiciones centrales (medidas a través del grado de intermediación y del prestigio de proximidad) se ajusta mejor para ambas redes y para ambas medidas en el caso de los expertos teóricos, tal vez porque sea más fácil percibir externamente que son "expertos" con respecto a los prácticos, tal vez porque no se considere que la experiencia internacional sea una forma de ser experto sobre asuntos políticos - téngase en cuenta que, como ya se explicó en el apartado metodológico, se pidió mencionar a personas con las que se habla de todo tipo de asuntos políticos. De hecho, la escala de experto teórico muestra correlaciones significativas y en algunos casos moderadamente altas con muchas medidas de centralidad usadas habitualmente.

2) Los expertos tienden a ocupar posiciones recompensadas socialmente, medidas a través de la escala de posición social de Galtung, aunque adaptada a la complejidad de la sociedad actual. Esta tendencia se confirma más claramente en la red primera que en la segunda, aunque debe tenerse en cuenta que, en general, los miembros de dicha red muestra puntuaciones ligeramente superiores a los de la segunda.

3) Los encuestados nombran principalmente a personas con las que tienen lazos fuertes en su red de discusión política, pero alrededor de una quinta parte de estas conversaciones se mantienen con personas con vínculos débiles, por lo que se comprueba que estas relaciones pueden ser importantes para difundir información novedosa. Estas personas son fundamentalmente amigos no íntimos. 


\section{Bibliografía}

Becker, M. H. (1970). "Sociometric Location and Innovativeness: Reformulation and Extension of the Diffusion Model"; American Sociological Review, no 35, Washington, pp. 267-282.

Berelson, B. R., Lazarsfeld, P. F. y McPhee, W.N. (1963). Voting. A Study of Opinion Formation in a Presidential Campaign; Chicago: University of Chicago Press.

Burt, R.S. (2001). "Structural Holes versus Network Closure as Social Capital", en Lin, N., Cook, K.S. y Burt, R.S. (eds.), Social Capital: Theory and Research; Nuev York: Aldine de Gruyter.

Coleman, J. et al (1966). Medical Innovation: A Diffusion Study; Nueva York: Bobbs-Merry.

De Nov, W., Mrvar, A. y Batagelj, V. (2004). Exploratory social network análisis with Pajek; Cambridge: Cambridge University Press.

Deutsch, K. W. (1952). Nationalism and Social Communication; Cambridge: MIT Press.

Díez Nicolás, J. (1992) "Posición social, información y postmaterialismo"; REIS, no 57, Madrid, pp. 21-35.

Díez Nicolás, J. (2005).; El dilema de la supervivencia. Los españoles ante el Medio Ambiente; Madrid: Obra Social Caja Madrid.

Galtung, J. (1964). "Foreign policy opinion as a function of social position"; Journal of Peace Research, 3-4, Oslo, pp. 206-231.

Granovetter, M.; "The Strength of Weak Ties" (1973). American Journal of Sociology; no 78, Chicago, pp. 1360-1380.

Granovetter, M.S. (1982). "The Strenght of Weak Ties. A Network Theory Revisited", en Peter V. Marsden y Nan Lin (eds.) Social Structure and Network Analysis, Beverly Hills: SAGE, pp. 105-130.

Harary, F., Norman, R. y Cartwright, D. (1965). Structural Models; Nueva York: Wiley.

Inglehart, R. (1977). The silent revolution: Changing Values and Political Styles among Western Publics; Nueva Jersey: Princeton University Press.

Inglehart, R. (1990). Cultural Shift in Advanced Industrial Society; Nueva Jersey: Princeton University Press.

Katz, E y Lazarsfeld, P.F. (1955). Personal influence. The part played by people in the flow of mass communications; Glencoe, Illinois: Free Press.

Lazarsfeld, P. F., Berelson, B. R. y Gaudet, H. (1954). The People's Choice. How the voter makes up his mind in a presidential campaign; Nueva York: Columbia University Press. 
Merton, R. K. (1949). Social theory and social structure; Glencoe, Illinois, Free Press. Edición empleada en este trabajo: Teoría y estructuras sociales; México D.F.: FCE.

Menzel, H. (1960). "Innovation, integration and marginality: A survey of pshysicians"; American Sociological Review, no 25, Washington, pp. 704-713.

Molina, J.L. (2001). El análisis de redes sociales. Una introducción; Barcelona: Edicions Bellaterra.

Requena Santos, F. (1996). Redes sociales y cuestionarios; Madrid: CIS, Cuadernos metodológicos, ํㅡ 18 .

Rogers, E. M. (1983). Diffusion of innovations; Nueva York: MacMillan.

Rogers, E.M., Ascroft, J.R. y Röling, N. (eds.) (1970). Diffusion of innovations in Brazil, Nigeria, and India; texto no publicado, Michingan, Michigan State University.

Rogers, E.M. y Kincaid, D.L. (1981). Communication networks: A new paradigm for research; Nueva York: Free Pres.

Valente, T. (1995). Network models of the diffusion of innovations; Nueva York: Hampton Press. 